\title{
Impacto de la terapia inhalada con $\beta-2$ agonistas de larga duración sobre la calidad de vida en pacientes asmáticos
}

\author{
O. VEGAZO GARCÍA, J. MARTÍN FERNÁNDEZ, C. BARCINA SÁNCHEZ, \\ F. J. JIMÉNEZ JIMÉNEZ, R. ESTIARTE NAVARRO
}

Departamento Médico. AstraZeneca Farmacéutica Spain. Madrid

IMPACT OF THE LONG ACTING BETA-2 AGONISTS INHALED
THERAPY ON THE QUALITY OF LIFE IN ASTHMATIC PATIENTS

\section{RESUMEN}

Objetivo: Evaluar el impacto sobre la calidad de vida relacionada con la salud (CVRS), de la adición de un $\beta_{2}$-agonista de larga duración inhalado (formoterol) en pacientes asmáticos en tratamiento con corticoesteroides inhalados, sin buen control.

Métodos: Estudio observacional, prospectivo, naturalístico de 6 meses de duración de una cohorte de 343 pacientes. Se recogieron variables sociodemográficas, clínicas y de función pulmonar. Se valoró la CVRS mediante una escala genérica (SF-36) y una específica (AQLQ de Marks).

Resultados: Se evaluaron 268 pacientes (78\%), con una edad media de 48,6 años (DE 16,28), el 64,15\% mujeres. Las mujeres y las personas con peor situación socioeconómica tenían basalmente una peor percepción de la CVRS. Durante el seguimiento se observó un incremento medio en el FEV1 de 0,26 1 (DE 0,44; p <0,001). El cambio medio en el AQLQ fue de -1,92 (-2,09-1,75), siendo más importante en el dominio de "ahogo". El cambio medio en el SF-36 fue de 5,13 (4,75-5,51) para la componente física estandarizada y de $5,23(4,83-5,63)$ para la componente mental. El tamaño del efecto fue "grande" con la medida del AQLQ y "moderado" con el SF-36. AQLQ y SF-36 se correlacionan estrechamente en sus puntuaciones, basales y tras el tratamiento $(r>0,5)$.

Conclusión: Añadir un $\beta_{2}$-agonista de larga duración inhalado (formoterol) al tratamiento de fondo de un paciente asmático mal controlado, en condiciones de práctica clínica habitual se asocia con una mejora significativa de su percepción de la CVRS medida con instrumentos genéricos y específicos.

PALABRAS CLAVE: Asma. Calidad de vida relacionada con la salud. Agonista beta adrenérgico. Tratamiento. Cuestionarios.

\section{ABSTRACT}

Objective: To assess the impact of the addition of inhaled therapy with long acting $\beta_{2}$-agonists (formoterol) on the health related quality of life (HRQL) in asthmatic patients treated with inhaled corticosteroids and poorly controlled.

Methods: Observational prospective naturalistic study with a cohort of 343 ambulatory patients during 6 months. We collected socio-demographic, clinical and lung function variables. The HRQL was assessed by means of a generic scale (SF-36) and a specific one (Marks's AQLQ).

Results: They were evaluated 268 patients (78\%). mean age 48.6 years (SD 16.28), 64.15\% women. Women and the subjects with worse socioeconomic situation had poor basal HRQL scores. During the follow-up an average improvement in FEV1 of $0.26 l$ (SD 0.44; $p$ $<0.001)$ was observed. The average change in the AQLQ was -1.92 (2.09-1.75). being the larger improvement in the breathlessness domain. The average change in SF-36 was 5.13 (4.75-5.51) for physical standardized component and 5.23 (4.83-5.63) for mental component. The effect size was "large" with the measurement of the AQLQ and "moderate" with $S F-36$. The $A Q L Q$ and $S F-12$ scores are strongly correlated. basally and after the treatment $(r>0.5)$.

Conclusion: To add long acting $\beta_{2}$-agonists to the asthmatic patients treated with inhaled corticosteroids and poorly controlled, under usual clinical practice conditions, is associated with an improvement of the $H R Q L$ perception measured by means of generic and specific instruments.

KEY WORDS: Asthma. Quality of life. Adrenergic beta-agonist. Therapies. Questionnaires.

Vegazo García O, Martín Fernández, J, Barcina Sánchez, C, Jiménez. Jiménez. FJ, Estiarte Navarro R. Impacto de la terapia inhalada con ß-2 agonistas de larga duración sobre la calidad de vida en pacientes asmáticos. An Med Interna (Madrid) 2004; 21: $272-278$.

\section{INTRODUCCIÓN}

El asma es una enfermedad crónica, con una prevalencia importante en los países industrializados, aunque con diferencias regionales, que también se observan en nuestro país (1). Cada vez existen más evidencias de que se está produciendo un aumento tanto de la prevalencia, como de la morbilidad asociada (2). Aunque también está aumentando el arsenal terapéutico del que se dispone, no parece que este hecho tenga el impacto esperado sobre un mejor control de la enfermedad.

Por tanto, el asma es un problema sanitario de primera magnitud, por la gran cantidad de población afectada y por la morbimortalidad que produce, a lo que habría que añadir el coste sanitario y social asociado a su manejo (3) y la afecta-

Trabajo aceptado: 22 de enero de 2004

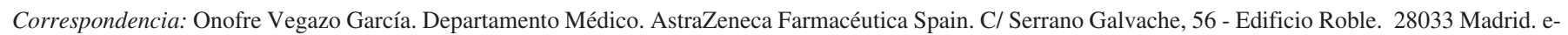
mail: Onofre.Vegazo@astrazeneca.com 
ción que supone sobre la calidad de vida y el bienestar de los pacientes $(4,5)$.

El pronóstico de un paciente asmático depende de un diagnóstico correcto, precoz y un apropiado manejo terapéutico, que incluye aspectos educativos y un trabajo específico sobre el uso de la terapia, que en general, se prolongará a lo largo de toda la vida. Los objetivos del tratamiento del asma se han ampliado más allá del control de los parámetros funcionales y los marcadores clínicos habituales de gravedad de la enfermedad e incluyen, actualmente, otras variables que contemplan las circunstancias individuales de cada paciente $(6,7)$.

Uno de los objetivos del tratamiento del asma es mejorar el bienestar del paciente, noción que incluye la mejora de la percepción que el paciente tiene de su enfermedad, y de su capacidad para desarrollar su vida diaria. Estos dos factores, percepción del paciente y capacidad funcional, están incluidos en el concepto definido por el término calidad de vida relacionado con la salud (CVRS) $(8,9)$, que en el caso del asma tiene una importancia especial.

La CVRS no se relaciona exclusivamente con la evaluación de la función pulmonar. De hecho, se ha observado que no existe una asociación fuerte entre las evaluaciones clínicas tradicionales de función pulmonar y las puntuaciones en CVRS (10,11). Esto ha llevado a muchos autores a señalar que, la medición de la CVRS, no puede inferirse de los parámetros de función pulmonar y se hace necesaria la utilización de escalas que permitan monitorizar su evolución en respuesta a los tratamientos tanto para la evaluación clínica en estudios de investigación como para la asistencia clínica habitual (12). Estas escalas han de permitir una valoración multidimensional y se acepta generalmente que deben incluir, al menos, cuatro dimensiones: física, funcional, psicológica y social.

Se diferencian dos tipos de escalas, las que permiten evaluar la CVRS en diferentes enfermedades, denominadas genéricas, y las específicas de patologías o sistemas. Se ha afirmado que las segundas son más sensibles a pequeñas modificaciones, relevantes clínicamente, en comparación con las genéricas (8).

Se considera que diferentes tratamientos, pueden tener diferente repercusión sobre la CVRS. En el caso de falta de control con corticoesteroides inhalados en pacientes con asma persistente, está recomendado el uso de $\beta_{2}$-agonistas de larga duración inhalados. Varios ensayos clínicos han demostrado la eficacia del formoterol, un $\beta_{2}$-agonista de acción prolongada y rápido inicio de acción, añadido a un corticoesteroide inhalado, tanto en el control de síntomas como en la mejora de la calidad de vida de pacientes asmáticos (13-15).

Dado que las condiciones de investigación clínica son, generalmente, rígidas en cuanto a los criterios de inclusión y exclusión, sin contemplar situaciones de comorbilidad o polimedicación, con unos niveles de cumplimiento terapéutico muy superiores a los habituales, y en unos grupos de edad muy concretos, no es sencillo para el clínico valorar la posible efectividad de un tratamiento, del que se conoce su eficacia, hasta que no se produce la fase de extensión de uso.

En este contexto se propone el presente estudio, cuyo objetivo es evaluar el impacto sobre la CVRS, de la terapia inhalada con un $\beta_{2}$-agonista de larga duración (formoterol) añadido al tratamiento de fondo, en una serie de pacientes asmáticos mal controlados, utilizando dos escalas de medición, una genérica y otra específica.

\section{MÉTODOS}

\section{PACIENTES}

Los pacientes asmáticos fueron reclutados en 20 consultas de neumología de la Comunidad de Madrid. Se incluyeron pacientes mayores de 18 años diagnosticados de asma, según criterios internacionales (GINA) (6), al menos 6 meses antes. Dichos pacientes debían haber seguido un tratamiento estable con corticoesteroides inhalados a dosis constante en las últimas cuatro semanas, necesitando utilizar agonistas $\beta_{2}$-adrenérgicos de acción corta de forma habitual para el control de sus síntomas y que, por indicación de su médico, fueran a iniciar tratamiento con formoterol, según las recomendaciones internacionales al uso.

Se excluyeron todos los sujetos que hubieran seguido tratamiento con agonistas $\beta_{2}$-adrenérgicos de larga duración previamente o que presentasen alguna contraindicación a los mismos.

\section{DISEÑO DEL ESTUDIO}

Se trata de un estudio observacional y prospectivo, naturalístico, con un seguimiento de 6 meses, de una serie de pacientes para evaluar el impacto del tratamiento con formoterol sobre la calidad de vida, en condiciones de práctica clínica habitual, mediante dos escalas, una genérica y otra específica de la enfermedad.

Durante el tiempo de seguimiento los pacientes fueron evaluados en cuatro ocasiones: basal, al mes, a los 3 y 6 meses.

\section{MEDICIONES}

En la visita basal se registraron las características sociodemográficas del paciente, edad, sexo, situación laboral, nivel de estudios y presencia de patologías concomitantes.

Durante el tiempo de seguimiento se hizo una monitorización de las pruebas de función pulmonar (FEV1 y FVC), de la situación clínica, las reacciones adversas y la calidad de vida. Para la determinación de la calidad de vida se utilizaron dos cuestionarios: SF-36 y AQLQ (Asthma Quality of Life Questionnaire). Esta monitorización tuvo lugar en cada visita.

El cuestionario SF-36 (16) es un cuestionario genérico autoadministrado que contiene 36 ítems agrupados en 8 dimensiones y 2 medidas globales: función física, limitación del rol físico, dolor corporal, percepción de salud general, vitalidad, función social, limitación del rol emocional, salud mental, componente física estandarizada y componente mental estandarizada. Para cada dimensión se obtienen valores comprendidos entre 0 y 100 siendo los más altos los indicativos de mejor calidad de vida.

El AQLQ de Marks (17) es un cuestionario específico de asma que consta de 20 ítems que hace referencia a la calidad de vida en las 4 semanas previas y se agrupan en 4 dimensiones: ahogo, estado de ánimo, restricción social y preocupación, además de una valoración global. Las puntuaciones toman valores comprendidos entre 0 y 10 y las puntuaciones más bajas indican mejor calidad de vida. 


\section{ANÁLISIS ESTADÍSTICO}

Las variables continuas se presentan como medias y desviaciones estándar (DE) y las cualitativas en forma de frecuencias absolutas y relativas.

Para las comparaciones de medias se utilizó el test de la t de Student (datos independientes y apareados) si las variables cumplían criterios de normalidad y el Signed Rank Test en caso de que las variables no siguieran este tipo de distribución. Para las comparaciones de variables cualitativas se utilizó el test de la chi cuadrado.

Las puntuaciones de las escalas de calidad de vida se correlacionaron con las características de los pacientes utilizando el coeficiente de correlación de Pearson (variables cuantitativas) y el test de Kruskal-Wallis (variables cualitativas).

Se calculó el tamaño del efecto para los cambios globales de la CVRS en cada escala dividiendo el cambio medio por la desviación estándar de los valores basales (18).

Para el análisis de los datos se utilizó SAS versión 8.0. El nivel de significación estadística para la comparación, se estableció en 0,05 .

\section{RESULTADOS}

Se incluyeron un total de 343 pacientes. De éstos, 75 $(21,9 \%)$ no completaron el seguimiento $(21$, el $6.1 \%$, abandonaron en el primer mes, 37, el 10,8\% antes de los tres meses, y 17 , el 5,0\%, antes de finalizar los seis meses). De los abandonos del protocolo, 5 se deben a efectos adversos $(1,5 \%)$. Finalmente, se analizaron 268 pacientes cuyas características basales se presentan en la tabla I. Se trata de una muestra con predominio de mujeres $(64,2 \%)$ y con una edad media de 48,6 años (DE 16,3). La función pulmonar basal mostraba un FEV1 medio de 2,18 1. (DE 0,96, rango 0,70-7,90)

La media de tiempo desde el diagnóstico de asma era de 10,5 años (DE 12,3). El 7,5\% de los pacientes fumaba en el momento de la inclusión, el 13,8\% había sufrido un ingreso en el último mes y el 50,4\% presentaban otra patología además del asma.

\section{MEDICIÓN BASAL DE LA CALIDAD DE VIDA}

La puntuación global basal con el AQLQ fue de 3,5 puntos (DE 2,1). Con el SF-36 fue de 43,0 ( DE 9,5) para la componente física estandarizada y de 45,6 (DE 10,7) para la componente mental estandarizado.

En el cuestionario específico, las dimensiones de calidad de vida que puntuaron de forma más negativa fueron las relacionadas con la sensación de ahogo y el estado de ánimo, mientras que en la escala genérica las dimensiones relacionadas con la percepción de salud general y la vitalidad fueron las peor valoradas.

La medición de la calidad de vida ofreció un perfil diferente en función de las características basales del sujeto. La valoración de la CVRS con el SF-36, en su componente física, mostraba unos valores peores en mujeres, en los pacientes hospitalizados en los últimos 12 meses, en los sujetos parados o retirados, los de más bajo nivel educativo, y en aquellos con patologías asociadas (Tabla II). Además se correlaciona posi-
TABLA I

CARACTERÍSTICAS BASALES DE LA POBLACIÓN SELECCIONADA PARA EL ESTUDIO

\begin{tabular}{|c|c|c|}
\hline Variables & $\%$ & Media $(D E)$ \\
\hline Edad (años) & & $48,6(16,3)$ \\
\hline \multicolumn{3}{|l|}{ Sexo } \\
\hline Hombres & 35,8 & \\
\hline Mujeres & 64,2 & \\
\hline Índice masa corporal $\left(\mathrm{kg} / \mathrm{m}^{2}\right)$ & & $26,7(4,5)$ \\
\hline Antigüedad diagnóstico (años) & & $10,5(12,3)$ \\
\hline$N^{o}$ hospitalizaciones último año & & $1,5(0,9)$ \\
\hline \multicolumn{3}{|l|}{ Hábito tabáquico } \\
\hline No fumador & 53,6 & \\
\hline Exfumador & 28,4 & \\
\hline Fumador ocasional & 10,5 & \\
\hline Fumador habitual & 7,5 & \\
\hline \multicolumn{3}{|l|}{ Situación laboral } \\
\hline Desempleado & 3,8 & \\
\hline Empleado & 43,9 & \\
\hline Ama de casa & 33,3 & \\
\hline Retirado & 10,8 & \\
\hline Estudiante & 8,2 & \\
\hline \multicolumn{3}{|l|}{ Nivel de estudios } \\
\hline Sin estudios & 5,6 & \\
\hline Estudios primarios & 39,0 & \\
\hline Estudios secundarios & 40,5 & \\
\hline Estudios universitarios & 15,0 & \\
\hline \multicolumn{3}{|l|}{ Cuestionario SF-36 } \\
\hline Función física & & $68,2(22,8)$ \\
\hline Rol físico & & $55,4(43,2)$ \\
\hline Índice de dolor & & $77,2(26,3)$ \\
\hline Percepción de salud & & $43,1(22,1)$ \\
\hline Vitalidad & & $52,8(20,1)$ \\
\hline Función social & & $70,5(26,1)$ \\
\hline Rol emocional & & $73,5(39,8)$ \\
\hline Índice de salud mental & & $62,3(19,7)$ \\
\hline $\begin{array}{l}\text { Componente física estandarizada } \\
\text { (global) }\end{array}$ & & $43,0(9,5)$ \\
\hline $\begin{array}{l}\text { Componente mental estandarizada } \\
\text { (global) }\end{array}$ & & $45,6(10,7)$ \\
\hline \multicolumn{3}{|l|}{ Cuestionario AQLQ } \\
\hline Sensación de ahogo & & $3,7(2,4)$ \\
\hline Estado de ánimo & & $3,6(2,5)$ \\
\hline Restricción social & & $3,3(2,6)$ \\
\hline Preocupación & & $3,3(2,3)$ \\
\hline Valoración global & & $3,5(2,1)$ \\
\hline
\end{tabular}

tivamente de manera moderada $(r=0,315)$ con la medida de la función pulmonar. La componente mental estandarizada era inferior en mujeres.

De forma parecida, la puntuación en la escala AQLQ se vio influida por la situación basal del paciente (Tabla III). Las personas desempleadas, sin estudios, y las que habían sido hospitalizadas en los últimos 12 meses tenían una peor puntuación global. Las mujeres además presentaban una peor percepción de la dimensión "estado de ánimo". Todas las puntuaciones se correlacionaban de manera negativa, aunque débil, 


\begin{tabular}{|c|c|c|c|}
\hline \multicolumn{4}{|c|}{ TABLA II } \\
\hline \multicolumn{4}{|c|}{$\begin{array}{c}\text { RELACIÓN ENTRE LAS CARACTERÍSTICAS BASALES DE LA } \\
\text { MUESTRA Y LAS PUNTUACIONES INICIALES DEL } \\
\text { CUESTIONARIO SF-36 }\end{array}$} \\
\hline & Correlación & $\begin{array}{l}\text { Puntuaciones } \\
\text { medias (DE) }\end{array}$ & $p$ \\
\hline \multicolumn{4}{|c|}{ Componente física estandarizada (Global) } \\
\hline Edad & $-0,028$ & & $<0,001$ \\
\hline IMC & $-0,207$ & & $<0,001$ \\
\hline \multicolumn{4}{|l|}{ Sexo } \\
\hline Hombre & & $44,3(9,8)$ & 0,020 \\
\hline Mujer & & $41,8(9,5)$ & \\
\hline \multicolumn{4}{|c|}{ Hospitalización últimos 12 meses } \\
\hline No & & $44,0(8,8)$ & $<0,001$ \\
\hline Sí & & $36,0(10,6)$ & \\
\hline \multicolumn{4}{|l|}{ Situación laboral } \\
\hline Desempleo & & $38,4(8,9)$ & $<0,001$ \\
\hline Empleado & & $45,0(8,7)$ & \\
\hline Ama de casa & & $40,9(9,5)$ & \\
\hline Retirado & & $37,8(11,7)$ & \\
\hline Estudiante & & $46,2(7,5)$ & \\
\hline \multicolumn{4}{|l|}{ Nivel de estudios } \\
\hline Sin estudios & & $38,1(11,4)$ & 0,003 \\
\hline Estudios primarios & & $41,6(8,8)$ & \\
\hline Estudios secundarios & & $43,0(10,0)$ & \\
\hline Estudios universitarios & & $47,0(8,0)$ & \\
\hline \multicolumn{4}{|l|}{ Patologías asociadas } \\
\hline No & & $44,1(8,6)$ & 0,002 \\
\hline Sí & & $39,8(10,6)$ & \\
\hline$F_{1}$ & 0,315 & & $<0,001$ \\
\hline FVC & 0,243 & & $<0,001$ \\
\hline \multicolumn{4}{|c|}{ Componente mental estandarizada (global) } \\
\hline \multicolumn{4}{|l|}{ Sexo } \\
\hline Hombre & & $47,5(10,6)$ & 0,039 \\
\hline Mujer & & $45,2(11,1)$ & \\
\hline
\end{tabular}

Se muestran sólo las variables con las que se encontró relación.

con las pruebas de función pulmonar. Tan sólo la dimensión "restricción social" no mostraba relación con ninguna de estas variables.

\section{EVOLUCIÓN DE LA CALIDAD DE VIDA DURANTE EL SEGUIMIENTO}

Las puntuaciones de la CVRS mejoran, utilizando ambos instrumentos de medida. Con la escala SF-36 se observa una mejoría estadísticamente significativa en todas las dimensiones de la calidad de vida y en las componentes física y mental globales, siendo las dimensiones de rol físico, rol emocional y la función social las que mayor mejoría experimentan (Tabla IV). Con el cuestionario AQLQ se observa un incremento en la calidad de vida en todas las dimensiones, especialmente marcada en la sensación de ahogo.

Al igual que las puntuaciones iniciales de la calidad de vida están influenciada por las características basales de los pacientes asmáticos, los cambios en la percepción de la calidad de vida también se relacionan con determinadas características.
TABLA III

RELACIÓN ENTRE LAS CARACTERÍSTICAS BASALES DE LA MUESTRA Y LAS PUNTUACIONES INICIALES DEL CUESTIONARIO AQLQ

$\begin{array}{cc}\text { Correlación } & \begin{array}{c}\text { Puntuaciones } \\ \text { medias }(D E)\end{array}\end{array}$

Nivel de estudios

Sin estudios

$4,0(2,5)$

0,018

Estudios primarios

$3,9(2,5)$

Estudios secundarios

$3,8(2,5)$

Estudios universitarios

$2,7(2,1)$

FVC

$-0,135$

0,018

\begin{tabular}{lll}
\hline \multicolumn{3}{c}{ Estado de ánimo } \\
\hline Sexo & $3,0(2,4)$ & 0,007 \\
$\quad$ Hombre & $3,8(2,6)$ & \\
Mujer & & \\
Hospitalización últimos 12 meses & $3,4(2,5)$ & 0,029 \\
$\quad$ No & $4,3(2,9)$ & \\
Sí & & \\
Situación laboral & $5,7(1,6)$ & 0,001 \\
$\quad$ Desempleo & $3,1(2,4)$ & \\
Empleado & $4,1(2,7)$ & \\
Ama de casa & $3,6(2,8)$ & \\
Retirado & $3,1(2,2)$ & \\
Estudiante &
\end{tabular}

Nivel de estudios

Sin estudios

$4,9(2,8) \quad<0,001$

Estudios primarios

$3,9(2,7)$

Estudios secundarios

Estudios universitarios

FEV

FVC

$-0,145$

$3,4(2,5)$

$2,4(1,9)$

0,012

$-0,238$

$<0,001$

\begin{tabular}{|c|c|c|c|}
\hline \multicolumn{4}{|c|}{ Preocupación } \\
\hline \multicolumn{4}{|c|}{ Hospitalización últimos 12 meses } \\
\hline No & & $3,0(2,3)$ & 0,014 \\
\hline Sí & & $4,0(2,7)$ & \\
\hline \multicolumn{4}{|l|}{ Nivel de estudios } \\
\hline Sin estudios & & $3,6(2,8)$ & 0,043 \\
\hline Estudios primarios & & $3,4(2,5)$ & \\
\hline Estudios secundarios & & $3,2(2,3)$ & \\
\hline Estudios universitarios & & $2,3(1,9)$ & \\
\hline FVC & $-0,139$ & & 0,015 \\
\hline \multicolumn{4}{|c|}{ Dimensión global } \\
\hline \multicolumn{4}{|c|}{ Hospitalización últimos 12 meses } \\
\hline No & & $3,3(2,1)$ & 0,020 \\
\hline Sí & & $4,2(2,5)$ & \\
\hline \multicolumn{4}{|l|}{ Situación laboral } \\
\hline Desempleo & & $4,9(1,2)$ & 0,044 \\
\hline Empleado & & $3,2(2,1)$ & \\
\hline Ama de casa & & $3,5(2,1)$ & \\
\hline Retirado & & $4,2(2,6)$ & \\
\hline Estudiante & & $3,1(2,2)$ & \\
\hline \multicolumn{4}{|l|}{ Nivel de estudios } \\
\hline Sin estudios & & $4,0(2,4)$ & 0,014 \\
\hline Estudios primarios & & $3,6(2,2)$ & \\
\hline Estudios secundarios & & $3,5(2,1)$ & \\
\hline Estudios universitarios & & $2,5(1,8)$ & \\
\hline FVC & $-0,149$ & & 0,010 \\
\hline
\end{tabular}

Se muestran sólo las variables con las que se encontró relación. 


\begin{tabular}{lccc}
\multicolumn{4}{c}{ TABLA IV } \\
\multicolumn{4}{c}{ MESES } \\
\hline SF-36 & Diferencia $^{a}$ & $I C 95 \%$ & $p$ \\
\hline Función física & 12,0 & $(11,4,12,5)$ & $<0,001$ \\
Rol físico & 28,4 & $(27,6,29,3)$ & $<0,001$ \\
Índice de dolor & 6,6 & $(6,0,7,1)$ & $<0,001$ \\
Percepción salud general & 9,2 & $(8,7,9,7)$ & $<0,001$ \\
Vitalidad & 11,8 & $(11,2,12,3)$ & $<0,001$ \\
Función social & 13,6 & $(13,0,14,3)$ & $<0,001$ \\
Rol emocional & 15,9 & $(15,1,16,7)$ & $<0,001$ \\
Índice salud mental & 9,4 & $(8,9,9,9)$ & $<0,001$ \\
Componente física & 5,1 & $(4,8,5,5)$ & $<0,001$ \\
Componente mental & 5,2 & $(4,8,5,6)$ & $<0,001$ \\
\hline TEST MARKS & Diferencia & $I C 95 \%$ & $p$ \\
\hline Sensación de ahogo & $-2,3$ & $(-2,4,-2,1)$ & $<0,001$ \\
Estado de ánimo & $-1,8$ & $(-2,0,-1,6)$ & $<0,001$ \\
Restricción social & $-1,8$ & $(-2,0,-1,7)$ & $<0,001$ \\
Preocupación & $-1,8$ & $(-2,0,-1,7)$ & $<0,001$ \\
Global & $-1,9$ & $(-2,1,-1,8)$ & $<0,001$ \\
\hline
\end{tabular}

aPuntuación SF-36 final menos puntuación SF-36 inicial

bPuntuación AQLQ final menos puntuación AQLQ inicial

Utilizando el SF-36 (Tabla V), ninguna de las variables que basalmente se relacionaban con una mejor percepción de la CVRS se asociaba con mejoras más significativas. Sólo la ausencia de patología concomitante se relacionó con un cam-

\section{TABLA V}

RELACIÓN ENTRE LA EVALUACIÓN EN LA CALIDAD DE VIDA Y CARACTERÍSTICAS BASALES DE LA MUESTRA*

\begin{tabular}{|c|c|c|c|}
\hline & Correlación & $\begin{array}{c}\text { Diferencias } \\
\text { medias }(D E)^{a}\end{array}$ & $p$ \\
\hline \multicolumn{4}{|c|}{ SF-36 Componente física estandarizada (global) } \\
\hline Edad & $-0,070$ & & 0,323 \\
\hline IMC & 0,018 & & 0,788 \\
\hline Sexo & & & 0,617 \\
\hline Hospitalización último & & & 0,435 \\
\hline Situación laboral & & & 0,505 \\
\hline Nivel de estudios & & & 0,402 \\
\hline Patologías asociadas & & & 0,414 \\
\hline $\mathrm{FEV}_{1}$ & 0,043 & & 0,523 \\
\hline FVC & $-0,048$ & & 0,479 \\
\hline \multicolumn{4}{|c|}{ SF-36 Componente mental estandarizada (global) } \\
\hline \multicolumn{4}{|c|}{ Hábito tabáquico } \\
\hline No fumador & & $4,9(9,6)$ & \\
\hline Exfumador & & $3,5(8,1)$ & \\
\hline Fumador ocasional & & $8,7(9,9)$ & 0,034 \\
\hline Fumador habitual & & $10,8(10,6)$ & \\
\hline \multicolumn{4}{|l|}{ Patologías asociadas } \\
\hline No & & $6,5(9,3)$ & 0,016 \\
\hline Sí & & $2,9(10,00)$ & \\
\hline
\end{tabular}

TABLA V (CONT.)

RELACIÓN ENTRE LA EVALUACIÓN EN LA CALIDAD DE VIDA Y CARACTERÍSTICAS BASALES DE LA MUESTRA*

\begin{tabular}{|c|c|c|}
\hline Correlación & $\begin{array}{l}\text { Diferencias } \\
\text { medias }(D E)^{a}\end{array}$ & p \\
\hline \multicolumn{3}{|c|}{ AQLQ de MARKS: Sensación de ahogo } \\
\hline Nivel de estudios & & 0,689 \\
\hline \multicolumn{3}{|l|}{ Patologías asociadas } \\
\hline No & $-2,6(2,2)$ & 0,005 \\
\hline Sí & $-1,7(2,5)$ & \\
\hline FVC & & 0,519 \\
\hline \multicolumn{3}{|c|}{ AQLQ de MARKS: Estado de ánimo } \\
\hline Sexo & 0,518 & \\
\hline Hospitalización últimos 12 meses & & 0,559 \\
\hline Situación laboral & 0,129 & \\
\hline Nivel de Estudios & 0,132 & \\
\hline \multicolumn{3}{|l|}{ Patologías asociadas } \\
\hline No & $-2,0(2,1)$ & 0,024 \\
\hline Sí & $-1,4(2,4)$ & \\
\hline FEV1 & & 0,728 \\
\hline FVC & & 0,102 \\
\hline \multicolumn{3}{|c|}{ AQLQ de MARKS: Restricción social } \\
\hline $\begin{array}{ll}\text { Edad } & 0,174\end{array}$ & & 0,014 \\
\hline $\begin{array}{l}\text { Patologías asociadas } \\
\text { No }\end{array}$ & & 0.001 \\
\hline Sí & $\begin{array}{l}-2,1(2,0) \\
-1,3(2,0)\end{array}$ & 0,001 \\
\hline
\end{tabular}

Hospitalización últimos 12 meses

Nivel de estudios

0,494

0,991

FVC 0,350

\begin{tabular}{lrc}
\hline \multicolumn{3}{c}{ AQLQ de MARKS: Dimensión g/obal } \\
\hline Edad & 0,019 \\
Hábito tabáquico & & \\
$\quad$ No fumador & $-1,8(1,8)$ & 0,049 \\
$\quad$ Exfumador & $-1,8(1,8)$ & \\
Fumador ocasional & $-2,9(1,8)$ & \\
$\quad$ Fumador habitual & $-1,7(1,3)$ & \\
Patologías asociadas & $-2,2(1,8)$ & $<0,001$ \\
$\quad$ No & $-1,4(1,7)$ & \\
Sí & 0,314 \\
Hospitalización últimos 12 meses & 0,400 & \\
Situación laboral & 0,541 & \\
Nivel de estudios & & 0,392 \\
FVC & & \\
\hline
\end{tabular}

* Se muestran las variables con las que se encontró relación en la medida basal del SF-36, y aquellas que no se asociaron a la puntuación basal, pero sí al cambio de esta.

aEvolución media en las puntuaciones: puntuación visita final - puntuación visita inicial

bio mayor en la componente mental. Esto mismo se aprecia en sujetos fumadores respecto a los no fumadores. Una mejoría más acusada en fumadores se veía también en el análisis de la percepción general del estado de salud, y vitalidad. La ausencia de otras patologías se encontraba asociada a mejoras más 
importantes en las dimensiones percepción general de salud, vitalidad, función social e índice de salud mental.

En todas las dimensiones del AQLQ, se encontró una mejoría más notable en los pacientes sin patologías asociadas, $\mathrm{y}$ en los fumadores en la medida resumen de la CVRS.

Durante este periodo (6 meses) se produjo una mejora media en el FEV1 de 0,26 1 (DE 0,44, p<0,001).

\section{CORRELACIÓN ENTRE LAS ESCALAS}

Se han correlacionado las puntuaciones globales del cuestionario AQLQ de Marks con las componentes física y mental del SF-36, tanto en el momento basal, como en la evolución. En la visita de inicio el coeficiente de correlación entre la puntuación global del AQLQ y el SF-36 fue de -0,611 para la componente física y $-0,567$ para la componente mental estandarizada. Los valores en la visita del mes 6 fueron de $-0,654$ y $-0,521$ para las mismas correlaciones (en todos los casos $\mathrm{p}<0,001)$. El signo negativo se debe a que al mejorar la CVRS aumenta la puntuación en el SF-36 y disminuye en el AQLQ de Marks.

\section{DISCUSIÓN}

La adición de un $\beta_{2}$-adrenérgico de larga duración (formoterol), al tratamiento de fondo de pacientes asmáticos, con corticoesteroides inhalados y pobremente controlados, se asocia con una mejora en la percepción de la calidad de vida en pacientes asmáticos

El estudio de la calidad de vida en el asma se está convirtiendo en un elemento más de la valoración del paciente en la práctica clínica diaria. Esto es debido a que el asma es una enfermedad que repercute de una manera muy directa sobre la actividad de los pacientes. La presencia de síntomas hace que la calidad de vida de los pacientes asmáticos se vea reducida de forma considerable y más aún, cuanto mayor sea la intensidad y duración de los síntomas. Esto produce una serie de interacciones de las esferas física y psíquica que limitará de manera importante la vida de estos pacientes. Así el clínico debe incorporar también la percepción del paciente, a la hora de hacer la evaluación del paciente. La medición del estado de salud en la práctica clínica habitual podrá contribuir a la prestación de una asistencia de mayor calidad en el paciente con enfermedad respiratoria (19).

Los $\beta_{2}$-adrenérgicos de larga duración, tienen un lugar establecido en el tratamiento de los pacientes asmáticos $(6,7)$. Algunos fármacos de esta familia, como el formoterol, tienen un inicio de acción muy rápido, comparable a la de los $\beta_{2}$ adrenérgicos de vida media corta (20), y aportan la ventaja de su posología, puesto que pueden ser utilizados en sólo dos administraciones diarias $(21,22)$. En el estudio que presentamos se observa que el tratamiento con formoterol se asocia con una mejora en los parámetros de calidad de vida y de los valores espirométricos.

La mejora de la función pulmonar $\left(0,261\right.$ de $\mathrm{FEV}_{1}$ de media), no es de la magnitud observada en los ensayos clínicos (13), pero aún así se acompaña de un cambio importante en la percepción de la CVRS. No está definido el mínimo cambio clínicamente importante en el cuestionario AQLQ, pero la variación de la puntuación global es del orden de 0,9 desviaciones estándar de la medida basal, lo que nos sitúa en un tamaño del efecto "grande" (18). Con el SF-36 las variaciones en las componentes estandarizadas son del orden de 0,5 desviaciones estándar de la medida basal, esto es, un cambio "moderado", lo que era previsible dada la menor "sensibilidad" de los instrumentos genéricos respecto a los específicos $(23,24)$. Sin embargo la correlación entre ambos era buena, y también como ya se había observado, más alta para el componente físico del SF-36 (23). Así pues, tendrá relevancia para el clínico incorporar la percepción del paciente, pues posiblemente sea más sensible a cambios señalados que las pruebas de función pulmonar.

En cuanto a la situación basal, existen determinadas características en la población que determinaron diferentes valoraciones de los pacientes con respecto a su calidad de vida. Ya se había descrito el efecto del género sobre la CVRS en pacientes asmáticos. Las mujeres tienen una peor percepción de su CVRS (25), que no se relaciona con una peor función pulmonar, especialmente en los grupos de jóvenes y mujeres maduras (26). En nuestro medio, y en población general, también se había demostrado (con el SF-36) que el ser mujer y el envejecimiento se correlacionan con un peor percepción de la CVRS. Ese efecto era más claro para las dimensiones físicas del SF-36 (27) . Otros factores como la situación laboral y el nivel de educación influyen en la percepción de la salud y de hecho en la salud misma. Ambos parámetros están relacionados con la situación socioeconómica y esta se ha mostrado como un factor predictor independiente de la CVRS en pacientes asmáticos (28). También era esperable que los pacientes con otras patologías asociadas, tuviesen menos margen de mejora en su percepción de la CVRS, aunque se controlen mejor sus síntomas asmáticos, porque probablemente su situación se vea influenciada por el resto de patologías, sobre las que la intervención no tiene peso. Esta circunstancia se ha evidenciado en otros estudios sobre CVRS en pacientes asmáticos. Cuantas más condiciones mórbidas se asocian al asma, peor es la percepción de la CVRS (29). Más complicada es la interpretación de la asociación del tabaquismo con una mejora más acusada en la CVRS. Se conoce que la percepción basal de su estado de salud en asmáticos fumadores es peor (30). Esta asociación no se encontró en nuestro caso, aunque podrían existir factores confusores como la edad, cuyo análisis no se ha realizado por exceder los objetivos de este estudio. De ser así no sería sorprendente que quien parte de una peor situación basal mejore más en su percepción de la CVRS.

La medición de la calidad de vida tanto en los ensayos clínicos como en la clínica habitual, está siendo una práctica cada vez más reconocida y extendida no sólo para la caracterización inicial del paciente sino además como una herramienta útil para el seguimiento del impacto de los tratamientos (12).

Los estudios que evalúan la respuesta a los tratamientos en condiciones reales de uso, son de gran utilidad para conocer el impacto que una determinada aproximación terapéutica pueda tener sobre el curso de una enfermedad y su correspondiente repercusión en la calidad de vida de los pacientes. Este tipo de diseños presentan, no obstante, algunas limitaciones. La principal objeción es la ausencia de un grupo control que permita adjudicar el efecto conseguido a la intervención estudiada. Así no se pueden descartar efectos de "regresión a la media" o el conocido como "efecto estudio" que hace que los sujetos experimenten mejoría por el hecho de ser observados. 
Sin embargo, no existen razones para pensar que los cambios en las puntuaciones medias en los cuestionarios de CVRS, en una cohorte de este tamaño, puedan deberse a efectos de regresión a la media, o a fluctuaciones ajenas a la verdadera situación de la calidad de vida percibida por el paciente. Además, en condiciones de práctica clínica, y sin estar comparando dos intervenciones equivalentes, la utilización de un grupo control presentaría muchos problemas.

Por otro lado, lo que constituye el principal valor de estos estudios, es la obtención de datos en pacientes que probablemente sean más representativos de los que acuden a las consultas médicas en nuestro medio. Suelen ser sujetos con perfiles heterogéneos, de otros grupos de edades, con otras patologías concomitantes, que necesitan de varios tipos de prescripciones farmacológicas, y con menores tasas de adherencia a los tratamientos, en definitiva no tan seleccionados como son los incluidos en los ensayos clínicos. De hecho, observando las características demográficas de los pacientes incluidos en el estudio, se comprueba que no difieren de las encontradas en otros estudios epidemiológicos (31). Además, los investigadores que participan en este tipo de estudios presentan un perfil más acorde con el resto de los profesionales de nuestro entorno. Consideramos que los datos mostrados pueden ser aplicables a la población de pacientes asmáticos que se atiende en las consultas de neumología de nuestro medio.

En definitiva, parece que añadir un $\beta_{2}$-agonista inhalado de larga duración (formoterol), al tratamiento de fondo de pacientes asmáticos, inadecuadamente controlados, se relaciona con una mejoría en la percepción de la calidad de vida. Además estos cambios se observan tanto con cuestionarios genéricos, como con cuestionarios específicos para el asma.

\section{Bibliografía}

1. Grupo Español del Estudio Europeo del Asma. Estudio Europeo del Asma. Prevalencia de hiperreactividad bronquial y asma en adultos jóvenes de cinco áreas españolas. Med Clin (Barc) 1996; 106: 761 - 767.

2. Wieringa MH, Vermeire PA, Brunekreef B, Weyler JJ. Increased occurrence of asthma and allergy: critical appraisal of studies using allergic sensitizacion, bronchial hyper-responsiveness and lung measurements. Clin Exp Allergy 2001; 31: 1553- 1563.

3. Barnes PJ, Jonsson B, Klim JB. The cost of asthma. Eur Respir J 1996; 9: 636-642.

4. Adams R, Wakefield M, Wilson D, Parsons J, Campbell D, Smith b, Ruffin R. Quality of life in asthma: a comparison of community and hospital asthma patients. J Asthma 2001; 38: 205- 214.

5. Juniper EF. Quality of life considerations in the treatment of asthma. Pharmacoeconomics.1995; 8: 123- 138.

6. NHLB/WHO Workshop Report: Global Strategy for Asthma Management and Prevention. National Institutes of Health. National Heart, Lung and Blood Institute. NIH Publication $\mathrm{N}^{\circ}$ 02-3659. Updated on 2002. In: http://www.ginasthma.com.

7. British Thoracic Society, Scottish Intercollegiate Guidelines Network. British guideline on the management of asthma. Thorax 2003; 58: (Supl. 1): 1-94.

8. Badía Llach X. La medida de la calidad de vida relacionada con la salud en los ensayos clínicos. Farmaeconomía: Evaluación económica de medicamentos 1995; 3: 51-76.

9. Schipper H, Clinch J, Powell V. Definitions and conceptual isssues. In: Spilker B, editor. Quality of life and pharmacoeconomics in clinical trials. Philadelphia:Lippincott-Raven Publishers, 1996: 11-23.

10. Juniper E, Guyatt GH, Ferrie PJ, Griffith LE. Measuring quality of life in asthma. Am Rev Resp Dis 1993; 147: 832-838.

11. Moy ML, Israel E, Weiss ST, Juniper EF, Dube L, Drazen JM. NHBLI Asthma Clinical Research Network. Clinical predictors of health-related quality of life depend on asthma severity. Am J Respir Crit Care Med 2001; 163: 924- 929.

12. Juniper EF. Using humanistic health outcomes data in asthma. Pharmacoeconomics 2001; 19 (Supl. 2): 13-19

13. Pauwels RA, Löfdahl CG, Postma DS, Tattersfield AE, O'Byrne P, Barnes PJ, et al. Effect of inhaled formoterol and budesonide on exacerbations of asthma. Formoterol And Corticosteroids Establishing Therapy (FACET) International Study Group. N Engl J Med 1997; 337: $1405-1411$.

14. O’Byrne PM, Barnes PJ, Rodriguez-Roisin R, Runnerstrom E, Sandstrom T, Svensson K, et al. Low dose inhaled budesonide and formoterol in mild persistent asthma: the OPTIMA randomized trial. Am J Respir Crit Care Med 2001; 164: 1392-1397.

15. Juniper EF, Svensson K, O’Byrne PM, Barnes PJ, Bauer CA, Lofdahl $\mathrm{CG}$, et al. Asthma quality of life during 1 year of treatment with budesonide with or without formoterol. Eur Respir J 1999; 14: 1038- 1043.

16. Alonso J, Prieto L, Antó JM. La versión española del SF-36. Heath Sur- vey (cuestionario de salud SF-36): un instrumento para la medida de los resultados clínicos. Med Clin (Barc) 1995: 104: 771-776.

17. Perpiñá M, Belloch A, Pascual LM y De Diego A, Comte L. Calidad de vida en el asma: validación del cuestionario AQLQ para su utilización en población española. Arch. Bronconeumol 1995; 31: 211-218.

18. Casado A, Prieto L, Alonso J. El tamaño del efecto en la diferencia entre dos medias: ¿estadísticamente significativo o clínicamente relevante? Med Clin (Barc) 1999; 112: 584-588.

19. Sanjuás C, Alonso J. Medir la calidad de vida relacionada con la salud en neumología: para qué y cómo. Arch Bronconeumol 1995; 31: 199201.

20. Cazzola M, Grella E, Matera MG, Mazzarella G, Marsico SA. Onset of action following formoterol Turbuhaler and salbutamol pMDI in reversible chronic airway obstruction. Pulm Pharmacol Ther. 2002; 15: 97102.

21. H. Moore R, Khan A, F. Dichey B. Long-acting inhaled b2 -Agonist in Asthma Therapy. Chest April, 1998; 113.4

22. Sears MR, Taylor DR, Print CG, Lake DC, Li Q, Flannery EM et al. Regular inhaled beta-agonist treatment in bronchial asthma. Lancet 1990; 336: 1391-1396

23. Mancuso CA, Peterson MG, Charlson ME. Comparing discriminative validity between a disease-specific and a general health scale in patients with moderate asthma. J Clin Epidemiol 2001; 54: 263-274.

24. Juniper EF, Norman GR, Cox FM, Roberts JN. Comparison of the standard gamble, rating scale, AQLQ and SF-36 for measuring quality of life in asthma. Eur Respir J 2001; 18: 38-44.

25. Leidy NK, Coughlin C. Psychometric performance of the Asthma Quality of Life Questionnaire in a US sample. Qual Life Res 1998; 7: 13734.

26. Wijnhoven HA, Kriegsman DM, Snoek FJ, Hesselink AE, de Haan M. Gender differences in health-related quality of life among asthma patients. J Asthma. 2003; 40: 189-199.

27. Alonso J, Regidor E, Barrio G, Prieto L, Rodríguez C, dela Fuente L. Valores poblacionales de referencia de la versión española del cuestionario de salud SF-36 Med Clin (Barc) 1998; 111: 410-416.

28. Apter AJ, Reisine ST, Affleck G, Barrows E, ZuWallack RL. The influence of demographic and socioeconomic factors on health-related quality of life in asthma. J Allergy Clin Immunol 1999; 103: 72- 78.

29. Wijnhoven HA, Kriegsman DM, Hesselink AE, de Haan M, Schellevis FG. The influence of co-morbidity on health-related quality of life in asthma and COPD patients. Respir Med. 2003; 97: 468- 475.

30. Gallefoss F, Bakke PS. Does smoking affect the outcome of patient education and self-management in asthmatics? Patient Educ Couns. 2003; 49: 91-97.

31. Barreiro E, Rubio M, Felisart F, Terrades J, Marcos R, Gra J. Características de los pacientes con asma bronquial atendidos de urgencia en un hospital de referencia de un área semirrural. Arch Bronconeumol 2000; 36: $172-179$. 